
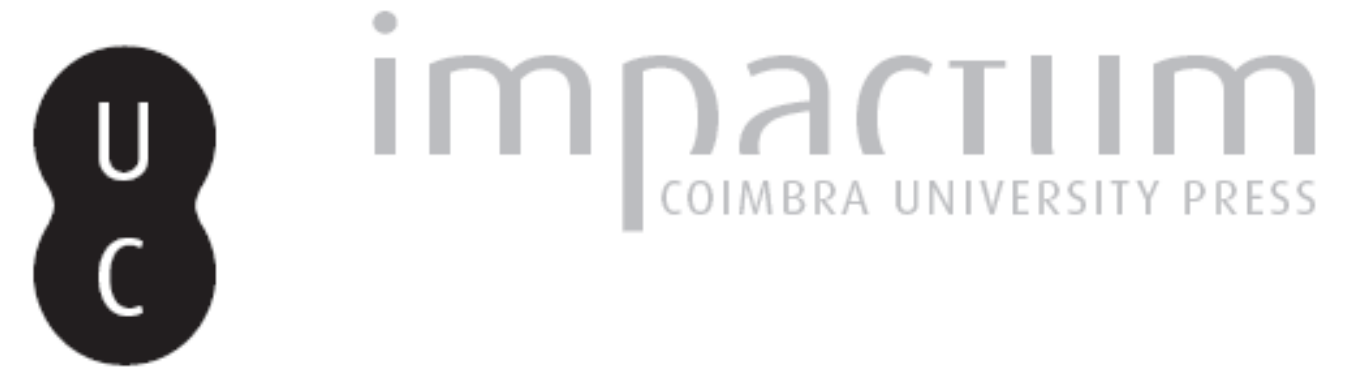

El epitafio poetico de L. Ivlivs Aptvs: (Mértola, Portugal)

Autor(es): Vicent, José Corell

Publicado por: Imprensa da Universidade de Coimbra

URL persistente: URI:http://hdl.handle.net/10316.2/45586

DOI: $\quad$ DOI:https://dx.doi.org/10.14195/1647-8657_27_3

Accessed : $\quad$ 26-Apr-2023 10:55:39

A navegação consulta e descarregamento dos títulos inseridos nas Bibliotecas Digitais UC Digitalis, UC Pombalina e UC Impactum, pressupõem a aceitação plena e sem reservas dos Termos e Condições de Uso destas Bibliotecas Digitais, disponíveis em https://digitalis.uc.pt/pt-pt/termos.

Conforme exposto nos referidos Termos e Condições de Uso, o descarregamento de títulos de acesso restrito requer uma licença válida de autorização devendo o utilizador aceder ao(s) documento(s) a partir de um endereço de IP da instituição detentora da supramencionada licença.

Ao utilizador é apenas permitido o descarregamento para uso pessoal, pelo que o emprego do(s) título(s) descarregado(s) para outro fim, designadamente comercial, carece de autorização do respetivo autor ou editor da obra.

Na medida em que todas as obras da UC Digitalis se encontram protegidas pelo Código do Direito de Autor e Direitos Conexos e demais legislação aplicável, toda a cópia, parcial ou total, deste documento, nos casos em que é legalmente admitida, deverá conter ou fazer-se acompanhar por este aviso. 
FACULDADE DE LETRAS

INSTITUTO DE ARQUEOLOGIA

\title{
CONIMBRIGA
}

\author{
VOLUME XXVII
}

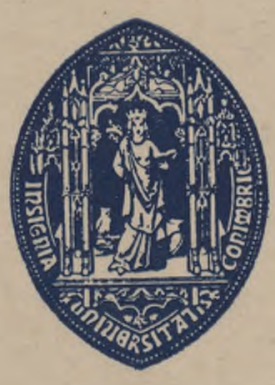

UNIVERSIDADE DE COIMBRA

1988 
Professor da Universidade de Valencia (Espanha)

EL EPITAFIO POETICO DE L. IVLIVS APTVS (MÉRTOLA, PORTUGAL) «Conimbriga», XXVII (1988), p. 141-151

RESUmo： Reexamina-se a problemática formal levantada por um epitáfio em verso proveniente de Mértola, no sul de Portugal. $\mathrm{O}$ autor dà nova leitura dalgumas das palavras, identifica numa das expressões uma passagem de Marcial e apresenta do texto uma interpretação mais correcta.

RÉSUMÉ : L'Auteur fait la révision — surtout du point de vue formel — des problèmes posés par un épitaphe métrique trouvé à Myrtilis (Conventus Pacensis), au sud du Portugal. Il y identifie un passage du poète latin Martial et présente, pour cela, une intérprétation plus correcte que les antérieures. 
(Página deixada propositadamente em branco) 


\section{EL EPITAFIO POETICO DE L. IVLIVS APTVS (MÉRTOLA, PORTUGAL)}

Del epitafio de L. Iulius Aptus, aparecido en Mértola y publicado por Vasconcelos en 1929, se han ocupado varios autores ${ }^{1}$ ). No obstante, considero oportuno volver sobre el mismo por dos razones fundamentalmente. En primer lugar, no se ha observado que el epitafio recoge una cita literal de Marcial. En segundo lugar, parece incorrecta la lectura que se ha hecho de la 1. 5. Se podría añadir también que no se ha hecho todavía un estudio detallado del epitafio desde el punto de vista filológico y literario.

Se trata de un ara de piedra caliza gris (mármol de Trigaches) con base y cornisa adornada con frontón y volutas $\left({ }^{2}\right)$. La rotura de

(9 José Leite de Vasconcelos, Epigrafia do Museu Etnológico [Belém), AP 28, 1927/29, 225 pp ss. núm. 35; ID., AP 29, 1930/31, p. 225; AE 1933, núm. 24; S. Mariner BigorRa, Inscripciones hispanas en verso, Barcelona-Madrid, 1952, p. 220 núm. 9; J. W. ZARker, Studies in the Carmina Latina Epigraphica, Princeton, 1958, núm. Ill; T. Scarlat Lambrino, Catalogue des inscriptions latines du Musée L. de V as concelo s, AP, III série, I, 1967, pp. 136 s., núm. 52 ; C. Magueiso, Uma inscrição métrica do Museu Nacional de Arqueologia e Etnologia, AP III série, IV, 1970, p. 116; J. Vives, Inscripciones latinas de la España romana, Barcelona, 1971/72, núm. 5814 (= ILEÏt); José D'ENCARNAÇão, Inscrições romanas do Conventus Pacensis. Subsídios para o estudo da romanização, Coimbra, 1984, pp. 160 ss. núm. 98 con fotografía; cf. J. M A NG As, Esclavos y libertos en la España romana, Salamanca, 1971, p. 333; P. Cugusi, Aspetti letterari dei Carmina Latina Epigraphica, Bolonia, 1985, p. 202.

(2) En la descripción del soporte y en las dimensiones del mismo, sigo a J. D'ENCARNAÇÂO, o.c., pp. $161 s$. 
la esquina inferior izquierda no afecta a la inscripción. Se conserva en el Museu Nacional de Arqueologia e Etnologia, de Lisboa, con el núm. E 6404.

Dimensiones: 94 X 45 X 33-36 cm.

Letras: 1. 1: 4,7 cm.; 1. 2: 3,5 cm.; 1. 3-6: $1,7 \mathrm{~cm}$.

El texto es (cf. fig. 1) :

L(ucio) Iulio Apto

Gallio patronus.

Itala me genuit tellus, Hispania texit.

Lustris quinque fui, sexta peremit hiemps.

5 Ignotus cunctis, hospes, qui hac sede iacebam, omnia qui nobis, hic dedit et tumulum.

Variantes: 1. 3 Hispaniae exii Vasconcelos, AP 28 1927/29; Hispania exil ILER; 1. 5 hospesque Vasconcelos, AP 29, 1930/31, Mariner, Zarker, Lambrino, Magueijo, ILER, Encarnação; 1. 6 onnia Vasconcelos, Mariner; o[m]nia Magueijo; omnia Encarnação; no[b]i[s] Vasconcelos, Mariner, Magueijo, ILER; tumulum Encarnação.

Se usa interpunción triangular en las 1. 1-2. La ordinatio del texto está muy cuidada: en el praescriptum, centrado y con letras de mayor tamaño que las de la parte poética, aparecen los nombres del homenajeado y del dedicante en las 1.1 y 2 respectivamente. Los cuatro versos han sido distribuidos cada uno en una línea y ordenados ostensiblemente en dísticos mediante el sangrado de los pentámetros. Las letras, estrechas y alargadas, se aproximan a la escritura actuaria. Por la tipología del monumento y la forma de las letras, puede datarse hacia finales del siglo $\mathrm{n}$ d. C. ( $\left.{ }^{3}\right)$.

El texto, como es habitual en las inscripciones poéticas, consta de dos partes: una en prosa, en la que figuran los nombres y la condición del homenajeado y del dedicante, la otra en verso.

(3) Esta es la datación que le atribuye J. D'ENCARnaÇA o, o.c., p. 162. 


\section{1-2: L(ucio) Iulio Apto / Gallio patronus.}

La condición de liberto de Aptus ( $\left.{ }^{4}\right)$, aunque no se indica en su nominación, resulta patente por el término patronus aplicado a Gallio, el dedicante $\left(^{5}\right)$. Este era, probablemente, de origen itálico, como su liberto, ya que el cognomen Gallio se encontraba extendido, sobre todo, en el norte de Italia $\left(^{6}\right)$. En Hispania tan solo aparece en otras dos inscripciones de Lusitania (GIL II 73, 323) y en una de la Bética (CIL II 2008).

El hecho de que los patronos honraran a sus libertos con epitafios fue muy frecuente desde los tiempos de la República hasta el siglo iY d. C. $\left({ }^{7}\right)$.

\section{3 : Itala me genuit tellus, Hispania texit.}

Obsérvese la triple aliteración a distancia (Itala... tellus... texit) y el sentido metafórico de texit 'cubrió', es decir, 'dio sepultura', en clara antítesis con genuit $\left({ }^{8}\right)$. Aparece aquí el tema de la muerte en ${ }^{*}$ lo* lo

(4) El cognomen Aptus es muy raro en Hispania. Aunque se trata de un cognomen adecuado para esclavos, no es significativo de status social, puesto que lo llevaban tanto personas de condición servil como libre; cf. I. KaJAnto, The Latin Cognomina, Helsinki, 1965, pp. 73, 134, 286; J. D'E NCAR N AÇÂ O, o. c. p. 161.

(5) J. Mangas, o. C., p. 333, considera erróneamente que Gallio forma parte de la nominación del liberto. En el mismo error incurre J. Vives, al registrar en los índices el nombre del difunto como L. Iulius Aptus Gallus, (ILER, pp. 699, 706).

(6) Cf. I. KaJanto, o. C., pp. 122,195; G. Alföldy, Die Personennamen in der römischen Provinz Dalmatia, Heidelberg, 1969, p. 209.

$\left.{ }^{7}\right)$ Cf. F. J. BRuns, Vier Kapiteln zu den Carmina sepulcralia latina in ihrer Eigenschaft aus Zeugnisse für das Lehen und Denken des kleinen Mannes der römischen Kaiserzeit, Diss., Göttingen, 1950, pp. 63 ss.; J. Mangas, o. c., pp. 247 ss.

$\left.{ }^{8}\right)$ Esta acepción poética de tegere es frecuente en los poetas clásicos (Tib., 3, 7, 204; Ver., A. 10, 904; Ov., 7>., 3, 3, 46; Prop., 1, 6, 28; Mart., 9, 29, 11, etc.) y, sobre todo, en los carmina epigraphica; cf. P. Cugusi, In margine al carmen epigraphicum del museo di S. Antioco (Sulci), «Epigraphica», 37, 1975, pp. 148 ss. 
un lugar distinto de aquel del nacimiento. Este tema se encuentra en más de 60 epitafios procedentes de las diversas áreas del Imperio $\left({ }^{9}\right)$. En Hispania mismo aparece cuatro veces más $\left({ }^{10}\right)$. Los destinatarios de tales epitafios son, generalmente, soldados, esclavos y libertos, comerciantes, marineros y viajantes $\left({ }^{\mathrm{n}}\right)$. El tema presenta diversas variantes, pero todas ellas tienen una estructura análoga: al lugar del nacimiento, indicado mediante gignere o algún sinónimo (nasci, creare, edere, alere, ferre, etc.) se suele oponer el lugar de la muerte y sepultura, indicando mediante tegere o afines contegere, retinere, rapere, tumulare, possidere, suscipere, recipere, tenere, etc.).

La gran difusión del tema parece explicarse por el deseo de morir y ser sepultado en el pais natal, tan arraigado en toda la Antigüedad clásica (12). Pudo contribuir también a su difusión el presunto autoepitafio de Virgilio, conocido ya desde finales del siglo i :

Mantua me genuit, Calabri rapuere, tenet nunc

Parthenope...

(9) Sobre este tema, identificado ya por F. BüCHELER, Carmina Latina Epigraphica, I-II, Leipzig, 1895/97, III Suplementum, ed. E. Lommatzsch. Leipzig, 1926 (= CLE), nùms. 474, 476, 478, 479 y 1266-268, véase E. Galletier, Étude sur la poésie funéraire romaine d'après les inscriptions, Paris, 1922, pp. 100 s.; H. Armini, Symbolae Epigraphicae, «Eranos» 26, 1928, pp. 256 ss.; P. Hoogma, Der Einfluss Vergils auf die Carmina Latina Epigraphica, Amsterdam, 1959, p. 221. P. Cugus I, o. c., ha estudiado el tema ampliándolo notablemente.

${ }^{10}$ ) En una inscripción de León (CLE 1526A), en otra de Córdoba (J. Mellado, J. M. Vila, Una inscripción romana hallada en Córdoba, «Habis» 3, 1972, pp. 321-324) y en dos, cristianas, la una de Braga y la otra de Hasta Regina (J. Vives, Inscripciones cristianas de la España romana y visigoda, Barcelona, 1962, núms. 275, 537).

(u) Cf. F. J. BRUNS, 。. C., p. 13; Cugusi, o. c., p. 217.

(12) El deseo de morir donde se ha nacido aparece tanto en la gran poesía (Verg., A. 5, 871; Ov., Tr. 3, 3, 45-46; Pont. 1, 2, 57-58; etc.) como en la epigrafía (CLE 800, 4; 1129, 2; 1312, 2-4; 1807A, 1 ; 2199; etc.) ; cf. A. Bréich, Aspetti della morte nelle iscrizioni sepolcrali dell'Impero romano, Budapest, 1937, pp. 36 s. ; R. Lаттімоге, Themes in Greek and Latin Epitaphs $s_{i}$ Urbana, 1942, pp. 200 ss.

(13) Sobre el epitafio de Virgilio, véase M. Betтini, L'epitafio di Virgilio, Silio Italico, e un modo di entendere la letteratura, «Dial. Archeol.», 9-10, 1976/77, 
La añoranza, pues, y el orgullo de Apto por su país natal, Italia, así como una probable alusión al epitafio de Virgilio pudieron inspirar este hermoso verso.

\section{4 : Lustris quinque fui, sexta peremit hiemps.}

Es habitual en los epitafios indicar la edad del difunto. Este dato, que constituye por lo generai una dificultad en la versificación, suelen aprovecharlo los poetas para hacer gala de su ingeniosidad $\left({ }^{14}\right)$. Pero, a veces, el poeta no sale airoso e incurre en la oscuridad, como en el caso que nos ocupa. Apto vivió cinco lustros, y el sexto invierno lo arrebató : la imprecisión es patente, tanto si el poeta ha dado a hiemps su acepción propia de 'invierno' como la poética de 'año' $\left({ }^{15}\right)$. Lo que quiere decir, sin duda, es que Apto murió en el primer invierno o año del sexto lustro. Pero hiems no tiene nunca dicho significado. La inexactitud se aclara, observando que el poeta ha tomado en préstimo un hemistiquio de uno de los tres epigramas que dedica Marcial a la niña Erotión :

Hic festinata requiescit Erotion umbra

crimine quam fati sexta peremit hiems

(Mart. 10,61,1-2) (16)

La expresión sexta peremit hiems es tan singular que no se puede pensar en una simple coincidencia. El hecho de que esa expresión resulte oscura, por no decir inadecuada, en el epitafio de Apto, confirma que se trata de un préstamo.

pp. 439 ss.; cf. también R. Chevalie R, Épigraphie et littérature à Rome, Faenza, 1972, p. 15 nota 38, pp. 53 s. ; P. Cugus I, o. C., p. 213 nota 15.

(14) En cuanto a la expresión de la edad en los epitafios, véase E. Galletier, o. C., pp. 255-259, 295; E. Liss Be RGe R, Das Fortleben der Römischen Elegiker in den Carmina Epigraphica, Diss. Tubinga, 1934, pp. 140-144; S. MARINER, o. C., pp.. 105-109.

$\left({ }^{15}\right)$ Hiems, en poesía, tiene con frecuencia la acepción de 'año'; cf. Thesaurus Linguae Latinae, VI, cols. 2778 s. (= TH LL).

(16) Véase también MaRT. 5, 34, 5; 5, 37, 16. 
La grafía hiemps, frecuente, aunque no atestiguada en los poetas clásicos $\left({ }^{17}\right)$, parece sugerir que nuestro poeta citaba de memoria.

No son nada frecuentes en la epigrafía hispánica las influencias de Marcial (18). Hasta ahora tan solo se habían observado en una inscripción de Tarragona, probablemente del siglo n (CLE 1279), y en otra de Sevilla, cristiana y del siglo vu (CLE 1392) $\left({ }^{19}\right)$.

\section{5 : Ignotus cunctis, hospes, qui hac sede iacebam.}

Vasconcelos, en un principio, propuso esta lectura, aunque traducía: «Neste territorio vivi como hospede, ignorado de todos» $\left({ }^{20}\right)$. Luego, por sugerencia de Wickert, corrigió el texto leyendo: Ignotus cunctis hospesque hac sede iacebam (21). Esta lectura, generalmente aceptada, parece incorrecta por varias razones. En primer lugar, hospes no se aplica nunca en los epitafios a los difuntos. Como se sabe, tanto en la epigrafía como en la gran poesía, es frecuente la eidolopoiía, figura consistente en presentar a los difuntos abordando a los vivos (22). Pues bien, uno de los términos más corrientes en tales casos es precisamente el de hospes. Este representa al lector casual del epitafio; es, por tanto, sinónimo

(17) Cf. ThLL col. 2773.

(18) Cf. S. Mariner Bigorra, Presencia de la poesía clásica en la España antigua, en «Simposio sobre la Antigüedad Clásica, Valle de los Caídos 1-3, XI, 1968», Madrid, 1969, pp. 127 s. Para la influencia de Marcial en los carmina epigraphica en general, véase H. I. Marrou, Deux inscriptions métriques d'Afrique, HEL 44, 1966, pp. 372 ss.; P. CuGUSi, Carmina Latina Epigraphica e tradizione letteraria, «Epigraphica», 44, 1982, pp. 99 s.; ID., O. C., pp. 190-194.

(19) Sobre la inscripción de Tarragona, véase P. Piernavieja Hozitis, Una nueva poesia de Marciai, «Emerita» 40, pp. 475 ss.; ID., Corpus de inscripciones deportivas de la España romana, Madrid, 1977, pp. 85 ss., 238; AE 1969/70, 275 bis; AE 1972, 283.

(20) AP 28, 1927/29, p. 227. La lectura hospes qui pasó a AE 1933, 24.

(21) AP 29, 1930/31, p. 225.

(22) Sobre la eidolopoiía, véase B. LIER, Topica carminum sepulcralium latinorum, «Philologus» 62, 1903, pp. 466 ss., 600 ss.; E. Galletier, o. C., pp. 38 Ss.; R. LATtimore, o. C., pp. 216-220, 230-237. 
de viator, lector, etc., tan frecuentes en las inscripciones (23). Por otra parte, sedes, en epigrafía, no significa nunca 'territorio', sino que es sinònimo de sepulcrum, tumulus, etc. $\left({ }^{24}\right)$. Finalmente, ¿acere no tiene en ningún epitafio el significado de 'habitar'. Su acepción es, generalmente, la de 'estar sepultado'. Lo que pudo inducir a error, aparte de que la lectura qui no aparece del todo nítida, es el imperfecto iacebam, que se creyó que haría referencia a la vida de Apto. Dicho imperfecto, en contraposición al perfecto dedit de la 1. 6, quizá sugiera que Apto, enterrado en un principio en una sepultura anónima, recibió después de su patrono un monumento con inscripción (25).

\section{6: Omnia qui nobis, hic dedit et tumulum.}

Omnia se lee con bastante claridad, a pesar de que la M, dada su estrechez, pudo dar la impresión a Vasconcelos de ser una $\mathrm{N}$ con el trazo interior al revés $\left({ }^{26}\right)$. Hic no es adverbio, sino pronombre y está en correlación con qui $\left.{ }^{27}\right)$. El paso de la primera persona singular, que aparece en las 1. 3-5, a la primera plural en la 1. 6 (nobis), es debido, sin duda, a necesidades métricas $\left({ }^{28}\right)$.

(23) Después de viator (unas 80 veces), el término más usado para abordar al transeunte es el de hospes (unas 55 veces); también son frecuentes lector, amice o perífrasis como tu qui praeteriens, tu qui legis, etc. ; cf. Concordance dei Carmina Latina Epigraphica, a cura di P. Golafrancesco e M. Massaro con la collaborazione di M. L. Ricci, Bari, 1986.

(24) Sobre la concepción del sepulcro como morada, véase E. GALLETIER, o. c., pp. 29 s., 255; R. Lattimore, o. C., pp. 165 ss., 318 s. Según F. A. Sullivan, Roman and Non-Roman in the Latin Epitaphs, TAPhA 70, 1939, p. 507, sedes, en el sentido de morada, aparece a partir del siglo ï a. C. y se usa en todos los periodos, incluso por los cristianos; al igual que domus, el término sedes se encuentra casi siempre en epitafios de personas de condición servil o de origen oriental.

(25) Cf. S. Marine R, o. с., p. 114.

(26) AP $28,1927 / 29$, p. 226. La grafía onnia por omnia y onnes por omnes tan solo aparece en algún que otro texto vulgar; cfr. ThLL IX, col. 609.

(27) VAsconcelos, AP 28,1927/29, p. 227, y J. EnCARnAÇ̃̃o, o. c., p. 160, ven en hic un adverbio.

(28) El plural poético no es frecuente en la epigrafía hispana y, por lo general, está condicionado por necesidades métricas; cf. S. MARINeR, o. c., pp. $91 \mathrm{~s}$. 
Por lo que se refiere a la estructura sintáctica, cada verso del primer dístico consta de dos oraciones independientes que se contraponen asindéticamente: a Itala me genuit tellus se opone Hispania texit, de la misma manera que a lustris quinque fui se contrapone sexta peremit hiemps. La estructura del segundo dístico consta de una oración compuesta, en la que a la subordinada de la 1. 5 se contrapone asindéticamente la oración principal de la 1. 6. La construcción sintáctica es un tanto relajada, pues presenta un nominativus pendens en la 1. 5. El autor incurrió en dicho anacoluto probablemente porque quiso poner de relieve no solo al destinatario del epitafio sino también a su dedicante.

En cuanto a la métrica, el epitáfio consta de dos dístico ${ }^{0}$ elegiacos prosódica y métricamente correctos. En el prime ${ }^{*}$ hexámetro, la $i$ breve de Itala se ha medido como larga por exigencias métricas. Pero, dada la corrección de toda la composición, no parece que esa escansión se deba a un error, sino más bien al conocimiento que el autor tenía de los poetas dactilicos, quienes frecuentemente miden como larga la $i$ breve de Italia y de sus derivados (Verg., A., 1, 2; 3, 185; 9, 698; Ov., Fast., 4, 64, etc.) En este hexámetro se combinam armoniosamente los dáctilos con los espondeos. Tiene cesura doble (trihemímera y heptemímera) y acaba en la cláusula $3+2$, la más frecuente tanto en los poetas clásicos como en la epigrafía. En el segundo hexámetro, todos los pies son espondeos, a excepción del quinto. \& Habrá querido el poeta expresar con ello la tristeza de quien yacía en un sepulcro anónimo ? La cesura es penthemímera y la cláusula $2+3$. Este hexámetro presenta en el tiempo no marcado del cuarto pie la elisión de qui. Pero, dado que se trata de una palabra con función puramente sintáctica, la elisión de este monosílabo, aunque poco frecuente, puede considerarse como normal $\left({ }^{29}\right)$.

En cuanto a los pentámetros, el primero finaliza con un bisílabo, mientras que el segundo lo hace con un trisílabo, evitado a partir de los poetas augústeos $\left({ }^{30}\right)$.

(29) Según J. Soubiran, Vélision dans la poésie latine, París, 1966, p. 405, el relativo qui en singular es elidido 17 veces en los poetas dactilicos.

( $\left.{ }^{30}\right)$ Cf. S. MarineR, o. C., p. 171. 
El análisis, pues, de la composición nos revela a un autor erudito. Conoce a Marcial y, probablemente, a Virgilio. Ciertos detalles, como la $i$ larga en Itala, la elisión en el pronombre qui, la métrica verbal, la aliteración Itala... tellus... texit, y la colocación de las palabras clave al inicio y al final de cada verso, confirman su erudición.

A la luz del anterior comentario, podría proponerse la siguiente tradución:

A Lucio Apto su patrono Galión. Itálica tierra me engendró, Hispania me ha dado sepultura. Viví cinco lustros, el sexto invierno me arrebató. En este sepulcro yacía, oh extranjero, desconocido de todos; mas quien me dio todas las cosas, me ha erigido también este monumento. 


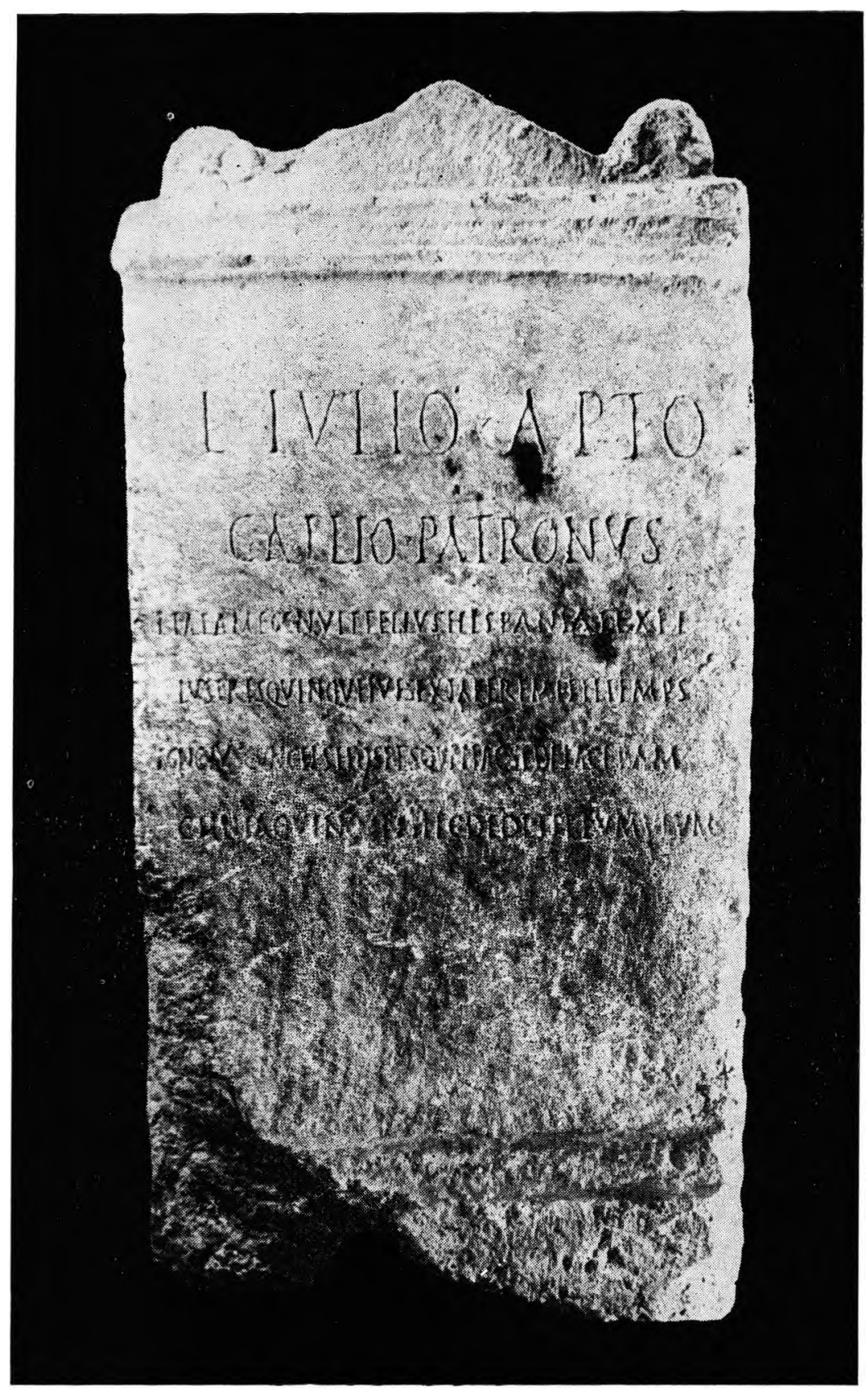

Foto de Guilherme Cardoso

F I G. 1 